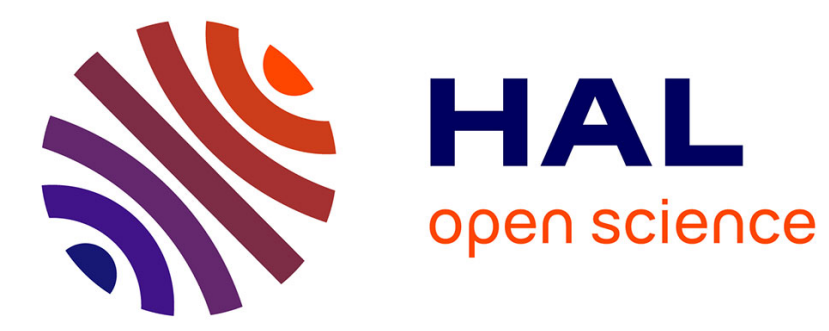

\title{
Comparison Of Methods Used For Computing The Impact Of Sound On The Marine Environment
}

Scott Schecklman, Dorian Houser, Matthew Cross, Dan Hernandez, Martin Siderius

\section{- To cite this version:}

Scott Schecklman, Dorian Houser, Matthew Cross, Dan Hernandez, Martin Siderius. Comparison Of Methods Used For Computing The Impact Of Sound On The Marine Environment. Marine Environmental Research, 2011, 10.1016/j.marenvres.2011.03.002 . hal-00703487

\section{HAL Id: hal-00703487 https://hal.science/hal-00703487}

Submitted on 2 Jun 2012

HAL is a multi-disciplinary open access archive for the deposit and dissemination of scientific research documents, whether they are published or not. The documents may come from teaching and research institutions in France or abroad, or from public or private research centers.
L'archive ouverte pluridisciplinaire HAL, est destinée au dépôt et à la diffusion de documents scientifiques de niveau recherche, publiés ou non, émanant des établissements d'enseignement et de recherche français ou étrangers, des laboratoires publics ou privés. 


\section{Accepted Manuscript}

Title: Comparison Of Methods Used For Computing The Impact Of Sound On The Marine Environment

Authors: Scott Schecklman, Dorian Houser, Matthew Cross, Dan Hernandez, Martin Siderius

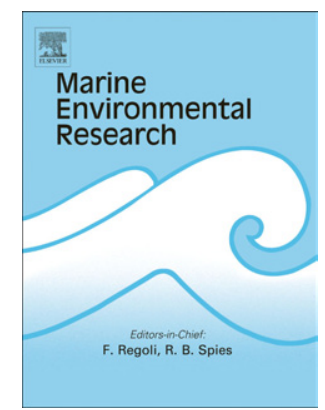

PII: S0141-1136(11)00043-2

DOI: 10.1016/j.marenvres.2011.03.002

Reference: MERE 3516

To appear in: Marine Environmental Research

Received Date: 10 May 2010

Revised Date: 30 March 2011

Accepted Date: 31 March 2011

Please cite this article as: Schecklman, S., Houser, D., Cross, M., Hernandez, D., Siderius, M. Comparison Of Methods Used For Computing The Impact Of Sound On The Marine Environment, Marine Environmental Research (2011), doi: 10.1016/j.marenvres.2011.03.002

This is a PDF file of an unedited manuscript that has been accepted for publication. As a service to our customers we are providing this early version of the manuscript. The manuscript will undergo copyediting, typesetting, and review of the resulting proof before it is published in its final form. Please note that during the production process errors may be discovered which could affect the content, and all legal disclaimers that apply to the journal pertain. 


\title{
Comparison of methods used for computing the impact of sound on the marine environment
}

\author{
Scott Schecklman ${ }^{\mathrm{a}}$, Dorian Houser ${ }^{\mathrm{b}}$, Matthew Cross $^{\mathrm{b}}$, Dan Hernandez ${ }^{\mathrm{c}}$, Martin Siderius ${ }^{\mathrm{a}, *}$ \\ ${ }^{a}$ Northwest Electromagnetics and Acoustics Research (NEAR) Lab, Portland State University PO Box 751 Portland, OR 97201-0751, USA \\ ${ }^{b}$ Biomimetica 7951 Shantung Dr. Santee, CA 92071, USA \\ ${ }^{c}$ HLS Research 3366 North Torrey Pines Court, Suite 310 La Jolla, CA 92037, USA
}

\begin{abstract}
Concern about the impact of sound on marine mammals has increased over the last decade, causing governments to take a more rigorous look at the potential impact of activities that introduce sound into the ocean. Environmental Impact Statements (EIS's) can be prepared using differing analysis methods to estimate the impact on marine mammals. To assess consistency in assessment methods, differences in the base assumptions were investigated; in particular, differences that arise between assumptions of dynamic marine mammals (animat method) and static distributions of marine mammals (static distribution method). Using several ocean environment scenarios and species, it is demonstrated that differences consistently arise between the two methods. The static distribution method underestimates the number of behavioral harassments compared with the animat method. Repeating many simulations with the animat method provides a robust risk assessment, provides a measure of variability, and allows the probability of "spurious events" to be estimated.
\end{abstract}

Keywords: Animat, Acoustic, Effects, Environmental impact, ESME, Mammal, Mathematical models, Navy sonar, Risk assessment

\section{Introduction}

The National Environmental Policy Act (NEPA) [42 U.S.C. 4321 et seq.] requires that all major federal actions conducted by the United States (U.S.) government consider the potential impact of the proposed action on the environment. When significant impacts to the environment are anticipated, or when there is significant public or scientific controversy over the impact of the proposed action, federal agencies are required to perform detailed assessments of the potential impact in an environmental impact statement (EIS). The development of an EIS for an action in the marine environment may also have to consider additional state and federal oversight of wildlife, water and air quality in the assessment. One such act, which has been at the center of litigation over the use of sonar by the U.S. Navy, is the Marine Mammal Protection Act (MMPA) [50 CFR 216].

Amendments to the MMPA in 1994 established and defined categories of impact to marine mammals resulting from human activity. The MMPA defines Level A

\footnotetext{
${ }^{*}$ Corresponding author: siderius@ @ pdx.edu
}

Preprint submitted to Marine Environmental Research harassment as, "any act of pursuit, torment, or annoyance which has the potential to injure a marine mammal or marine mammal stock in the wild." It further defines Level B harassment as, "any act of pursuit, torment, or annoyance which has the potential to disturb a marine mammal or marine mammal stock in the wild by causing disruption of behavioral patterns, including, but not limited to, migration, breathing, nursing, breeding, feeding, or sheltering but which does not have the potential to injure a marine mammal or marine mammal stock in the wild." These definitions were later modified for military readiness activities in the National Defense Authorization Act (2004). However, regardless of the definition employed, the MMPA requires that harassment be quantified in an EIS that involves the potential to affect marine mammals.

One of the first major EIS's undertaken by the U.S. Navy and which addressed the MMPA with respect to the potential impact of anthropogenic sound was the SEAWOLF shock-test (U.S. Department of the Navy, 1998), a test in which large underwater detonations were sequentially placed in closer proximity to a new class of submarine in order to determine the integrity of the

March 30, 2011 
vessel design. Controversy existed over this action not only with respect to the potential to injure marine mammals directly by exposure to blast waves, but over the potential for acoustic trauma to occur as well. Since that time, the use of sonar for military purposes has come under increasing scrutiny and speculation regarding its potential as a causative factor in atypical beaked whale strandings (Cox et al., 2006; Fernandez et al., 2005; Frantzis, 1998; Jepson et al., 2003; Rommel et al., 2006; Simmonds and Lopez-Jurado, 1991). Both mid-frequency $(1-10 \mathrm{kHz})$ and low-frequency $(<1 \mathrm{kHz})$ sonar have been targeted in the speculation; however, since an atypical beaked whale stranding event in the Bahamas in 2000 that coincided in time and space with a multi-ship mid-frequency active (MFA) sonar exercise (U.S. Department of Commerce and U.S. Department of the Navy, 2001), attention has predominantly focused on the potential for MFA sonar to impact marine mammals. The debate over the relationship between MFA and beaked whale strandings intensified with atypical beaked whale strandings that coincided with the use of MFA sonar in the Canary Islands (Fernandez et al., 2005; Jepson et al., 2003) and putative relationships between MFA sonar and beaked whale strandings in Madeira (D'Amico et al., 2009; Cox et al., 2006). Not surprisingly, concern about the impacts of MFA sonar on other marine mammals, and marine life in general, has continued to grow in light of these stranding events. It has expanded beyond concern for injury to the potential for alterations in behavior that are "biologically significant" (National Research Council, 2005).

Concomitant with an increased controversy over the impact of MFA sonar to marine mammals was a realization that US Navy at-sea ranges used for MFA sonar training and anti-submarine warfare (ASW) exercises had not achieved NEPA compliance with respect to the potential impact of MFA sonar on the environment. Furthermore, a new Surveillance Towed Array Sensor System-Low Frequency Active (SURTASS LFA) sonar, garnered enough public attention that an EIS governing its use was also deemed necessary prior to its deployment (U.S. Department of the Navy, 2001). In recent years, additional acoustic sources have been added to the list of items that must be considered as to their potential impact to marine mammals. As a result, there has been a dramatic push to complete EIS's for existing Navy ranges with a particular emphasis on the potential impacts of the numerous sources that may be deployed on these ranges.

Each EIS developed by the US Navy for estimating impacts to marine mammals resulting from sound exposure requires that an estimate of the number of Level
A and Level B harassments is made. Over the last decade this has been an evolving process; the criteria and thresholds for acoustic exposures equating to harassment have changed in response to new science and, where science is lacking, as a result of policy decisions. Complicating the issue is the fact that no definitive or standardized modeling approach has been established for determining the number of Level A or Level B harassments experienced by marine mammals as a result of sound exposure. The approach to modeling has typically been the decision of the action proponent and modeling approaches across the various EIS's have often employed different assumptions. For example, the original approach to modeling the impact of sonar on marine mammals for a proposed Undersea Shallow Water Training Range assumed that animals were static in the environment and that the exposure to sound emitted from a sonar system was "worst case" for any depth in the water column, i.e. it did not matter where the animal was in the water column, the maximum exposure was assumed (U.S. Department of the Navy, 2005). This approach was conservative in that it reduced the problem to a 2-dimensional space and it accepted that the level of impact would be overestimated.

In contrast, the Hawaii Range Complex (HRC) EIS assumed both a vertical (i.e. depth) and horizontal distribution of animals, the former of which was based upon the known or estimated dive profiles of the marine mammal (U.S. Department of the Navy, 2008). This 3-dimensional approach accounted for the probable occupation of a water depth by a marine mammal and permitted the sonar sound field, which can vary dramatically in the vertical plane, to be more accurately accounted for.

The third approach, utilized in the SURTASS-LFA EIS (U.S. Department of the Navy, 2001), was the use of animats, or artificial representations of marine mammals that move through a simulated environment in both time and space and account for the 4th dimension of the problem, i.e. time. This assessment relied heavily on the Acoustic Integration Model to integrate animat behavior with simulated sound exposures (Frankel et al., 2002).

Each of the methods used by the U.S. Navy for estimating impacts to marine mammals exposed to sound from Navy sources results in different levels of conservativeness and variability in impact estimates. Without adequate acknowledgment of the assumptions that are made in each of these processes, misunderstandings and confusion over impact estimates that are made across the various Navy EIS's can occur. Therefore, it would be beneficial to document the 
expectation of model outputs from each of the methods employed for estimating impacts to marine mammals arising from exposure to U.S. Navy sound sources. The quantification of the differences and an understanding of the reasons underlying the differences are important to providing a more stable interpretive framework for action proponents, regulatory agencies, and the legal system that must judge the adequacy of an EIS if brought to trial. Within this paper we address two of the base methods of modeling impacts to marine mammals that have been historically implemented. The core to each modeling approach is applied to several scenarios; post-analysis correction factors are not applied (e.g. as documented in Appendix $\mathbf{J}$ of the HRC EIS). Results are presented in context of expected variations in the modeling output and the discussion focuses on the relevance of the results to the real-world environment.

\section{Materials and methods}

\subsection{Harassment criteria and thresholds}

In this article we will consider only narrow band MFA sources such as those typical for Navy MFA sonars. For odontocete cetaceans (toothed whales), the U.S. Navy currently implements three sets of criteria and thresholds for estimating Level A and Level B harassment resulting from exposure to MFA sources (U.S. Department of the Navy, 2008). The criterion for a Level A take is considered to be the onset of Permanent Threshold Shift (PTS), or a permanent reduction in hearing sensitivity. Two criteria exist for Level B takes. The first criterion is the onset of a Temporary Threshold Shift (TTS), or a temporary reduction in hearing sensitivity (i.e. the "rock concert" effect). The logic behind using this as criterion for Level B harassment is that a reduction in hearing sensitivity, which is fully recoverable, may nevertheless impede the reception of sound that would normally alter the animal's behavior (e.g. sounds produced by conspecifics or predators). The second criterion is a behavioral response, in the absence of auditory fatigue, which is a significant alteration of a species' normal behavioral patterns.

Thresholds for harassment determined by the onset of PTS and TTS are based on the sound exposure level (dB re: $1 \mu P a^{2} \cdot s$ ) of an animal accumulated over the duration of an exercise involving MFA sources. Sound exposure level is defined as,

$$
S E L=10 \log _{10}\left(\frac{\int_{0}^{T} p^{2}(t) d t}{P_{r e f}^{2} T_{r e f}}\right)
$$

where $P_{r e f}=1 \mu \mathrm{Pa}$ and $T_{r e f}=1 \mathrm{~s}$. Level B harassment due to a behavioral alteration (i.e. in the absence of auditory fatigue) occurs when the maximum sound pressure level (SPL) received over the duration of the exercise is great enough to cause the mammal to significantly alter its normal behavioral patterns. Sound pressure level is defined in terms of the root-mean-squared pressure field $P$ as,

$$
S P L=10 \log _{10}\left(\frac{P_{r m s}^{2}}{P_{r e f}^{2}}\right)
$$

where $P_{\text {ref }}=1 \mu \mathrm{Pa}$.

For simplicity, the modeling and discussion in this article will be limited to Level B harassment due to behavioral disruption and in the absence of auditory fatigue. It will henceforth be referred to only as Level $\mathrm{B}$ harassment, or harassment. The number of harassments estimated to occur from an exercise involving MFA sources is calculated by evaluating a risk function (defined in Appendix $\mathrm{J}$ of the HRC EIS) that relates the risk of harassment to the maximum sound pressure level received by a marine mammal (U.S. Department of the Navy, 2008). The risk function varies between 0 (no risk) and 1 (maximum risk) and can be interpreted as the probability that an individual may alter its behavior in response to a given max SPL. It is subsequently interpreted as the proportion of the population that would be taken under identical exposure conditions, and is summed with all other risk function outputs to determine the number of harassments for an exposure scenario. This value can then be extrapolated to the population level.

The risk, $R$, is given by

$$
R=\frac{\left(\frac{L-B}{K}\right)^{A}}{1+\left(\frac{L-B}{K}\right)^{A}}
$$

where $L$ is the maximum received SPL level $(\mathrm{dB})$. The basement value, $B$, is $120 \mathrm{~dB}$, and $\mathrm{K}$ is set to 45 $\mathrm{dB}$ so that a received level of $165 \mathrm{~dB}$ corresponds to a $50 \%$ probability of harassment. We note that the algebraic form of the risk function in the literature (U.S. Department of the Navy, 2008) has been recast into the form shown in Equation 3 in order to avoid a divide-byzero error that would occur in the original formula when $L-B=K$. The risk transition sharpness parameter, $A$, depends on the marine mammal classification. For all odontocetes (toothed whales, dolphins and porpoises), except the harbor porpoise (Phocoena phocoena), $A=$ 10. (The harbor porpoise is given special consideration 


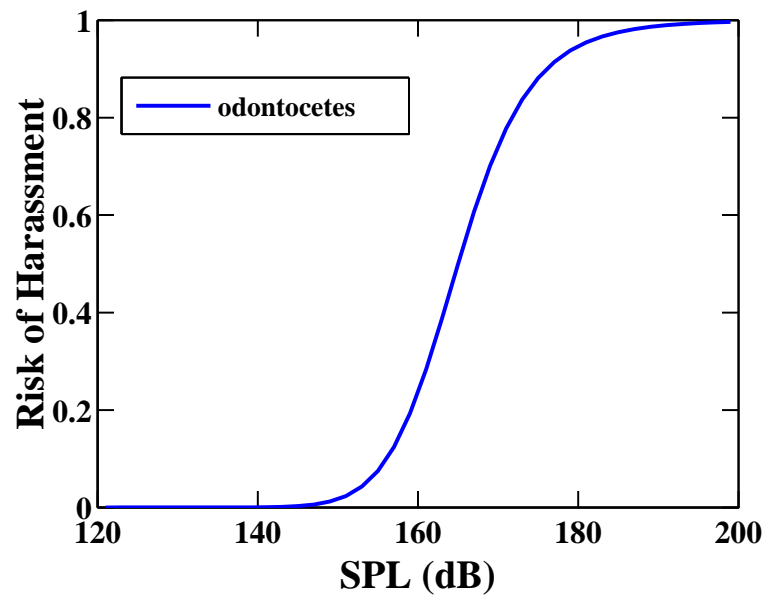

Figure 1: The risk function indicates the proportion of a population that is expected to alter its behavior in response to a given maximum received SPL. The risk of harassment rises sharply when the SPL approaches 160 $\mathrm{dB}($ re: $1 \mu \mathrm{Pa})$.

as a particularly sensitive species.) The risk function for odontocetes is plotted in Figure 1.

When the maximum received SPL is near $120 \mathrm{~dB}$, the risk of harassment is low. The risk rises sharply as the maximum received SPL approaches $160 \mathrm{~dB}$ and reaches a maximum value of 1 when the maximum received SPL is $195 \mathrm{~dB}$.

\subsection{Description of methods}

In this section we compare the dynamic animat method of distributing marine mammals with the 3dimensional static distribution method (hereafter referred to as the animat method and the static distribution method, respectively). Both methods were briefly described in the introduction but more details are given here with respect to how they are implemented in this comparison.

The animat method is a full 4-dimensional simulation of marine mammal dive behavior that can be considered as one of the individual based model variants (DeAngelis and Gross, 1992; Houser, 2006). The animats are dynamic entities with behavioral states governed by 1st order transition probabilities. Diving behavior within a behavioral state (e.g. deep foraging diving, shallow transit diving) is determined by a stochastic sampling of distributions that describe the various aspects of diving for that behavioral state (e.g. ascent and descent rate, maximum dive depth, surface interval time, etc.). Parameterization of the behavioral states is based on the current state of knowledge of the species in question. The animat method begins with a distribution of individual animats within the 3-dimensional ocean volume. The distribution for a given animat "species" is determined by the population density for the species being modeled and the dive distributions resulting from the initialized behavioral state. For the duration of the exercise, animats move dynamically through the simulated environment as governed by their behavioral state. The animat positions in range, bearing and depth are recorded over the duration of the exercise. At the conclusion of the exercise, the maximum received SPL resulting from each animat's movement through the simulated sound field is determined. Then, for each animat, this value is used in the risk function (Equation 3) to determine the proportion of the population that would be harassed during the exercise. Thus, only a fraction of each animat is harassed. The total number of harassments for an exercise is then determined by summing the risk score across all of the animats in the simulation.

The static distribution method begins with the same horizontal population density that is used in the animat method, but then uses a depth histogram to distribute the population density vertically in the water column. In effect, the ocean is divided into sub-volumes into which a fraction of the animal population is assumed to occur independent of time. Historically, depth histograms have been derived from the published literature. However, for the comparisons in this paper, the depth histogram is derived from the dive profiles of all of the animats run in the animat simulations. This permits some degree of equivalence between the methods in that the vertical distribution of animals is the same. However, whereas the animat method capitalizes on sound reception at individual animals, the static distribution method relies on the fractional distribution of animals that are nonmoving. Following the distribution of the animals, the transmission loss from the sound source is calculated and the maximum SPL received at each sub-volume of the ocean is determined (see below). These values are compared to the risk function to determine the risk for the sub-volume and then multiplied by the fraction of the animal population assigned to that volume. The summation of this product across all sub-volumes yields the total number of harassments.

The sound pressure field varies with range and depth potentially producing surface ducts or convergence and shadow zones (Urick, 1975). This suggests that some proportion of the animal population will exist "outside" of the convergence zones (or ducts) and receive relatively low SPL exposure, whereas a different proportion 
of the population will exist "inside" the convergence zones for the duration of the simulation. However, since harassment is determined by the maximum SPL at a given location in the ocean volume, the harassment for any given sub-volume will be a fixed value dependent on the maximum SPL determined for that volume and the proportion of the population that occupies it. That is to say, the estimate lacks variability. In the animat approach, an individual mammal is allowed to move through the simulation space and therefore pass in and out of a surface duct or convergence zone. This adds variability to the harassment estimate and can result in higher estimates due to animats traversing through multiple ocean sub-volumes (see below).

\subsection{Comparison example}

The following section provides a simple example to illustrate the differences between the static distribution and animat analysis methods. We begin by considering a 2-dimensional simulation space and calculating the number of harassments for a small population of animals by each of the methods. The simulation space is divided into two depth bins and two range columns, as shown in Figure 2. We assume that an underwater sound source has a source level (SL, in $\mathrm{dB}$ re: $1 \mu \mathrm{Pa}$ ) sufficient to produce a sound field with an SPL of 195 dB inside a surface duct (top depth bin in Figure 2). Below the surface duct (bottom depth bin in Figure 2 ) the SPL is $120 \mathrm{~dB}$. The sonar emits 2 pings, each with duration of 0.49 seconds. Thus, the total SEL is just below $195 \mathrm{~dB}$ and we do not have to consider harassment due to the onset of TTS. Two animats are in the first range column during the first ping and they are both in the second range column during the second ping. However, between pings, the second animat moves down below the surface duct.

According to the animat method, both animats experienced a maximum SPL of $195 \mathrm{~dB}$ at some point in the simulation. Applying the risk function to this maximum SPL we find there is a $100 \%$ chance that animat \#1 will be harassed (during either the first or second ping). Likewise, from the risk function, there is a $100 \%$ chance that animat \#2 will be harassed (due to the first ping). Thus, a total of 2 harassments are predicted to occur as a result of the scenario.

In the static distribution method the total number of animats is first distributed among the range columns. Since, there are two animats in the simulation space and two range columns; we will have on average 1 animat per range column. Next, we consider the animal depth

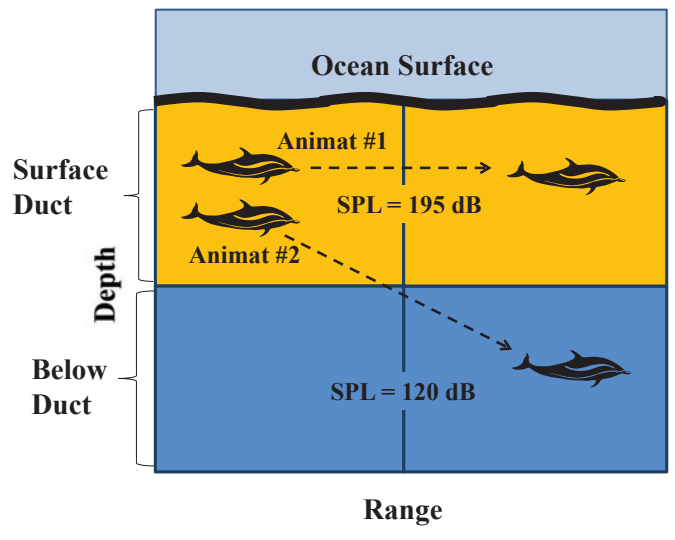

Figure 2: Illustration of the dive path for two animats in a simple 2 dimensional ocean environment containing a surface duct (shown in orange). The animat method considers both animats to be harassed due to their initial exposure to an SPL of $195 \mathrm{~dB}$. For the static distribution method the space is divided into 4 quadrants; 2 range columns and 2 depth bins. The movement of animat \#2 below the surface duct results in the depth histogram shown in Figure 3.

distribution from the dive patterns shown in Figure 2. The animats occupy 4 depths across 2 time steps: 3 in the top depth bin (surface duct) and 1 in the lower depth bin (below the surface duct). The resulting depth histogram for this hypothetical species is shown in Figure 3.

The depth distribution is then multiplied by the density of the range columns and the results are added together to get the total number of harassments, $H$. The formula used for each quadrant is,

$$
H=\left(\frac{N}{C_{r}}\right) \times D \times R
$$

where $N$ is the total number of animats in the simulation space, $C_{r}$ is the total number of range columns, $D$ is the depth bin distribution (taken from figure 3 ), and $R$ is the risk from Equation 3.

In the upper left quadrant the number of harassments is, $H=(2 / 2) \times 0.75 \times 1=0.75$, and in the bottom left quadrant we have $H=(2 / 2) \times 0.75 \times 0=0$, for a total of $0.75+0=0.75$ harassments in the left range column. Likewise, for the range column on the right side we have the identical results. Thus, the static distribution method arrives at a total of $0.75+0.75=1.5$ harassments in this simulation space. We note that this is $25 \%$ 


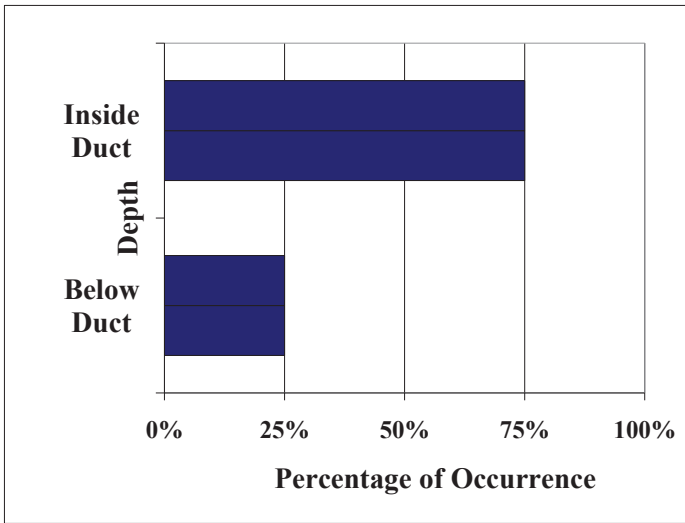

Figure 3: The depth histogram for the static distribution analysis of the animats in Figure 2 places $3 / 4$ of the animats in the top depth bin and 1/4 in the lower depth bin. The permanent placement of $25 \%$ of the animats below the surface duct results in $25 \%$ fewer harassments in this simulation method.

less than the number of harassments determined using the animat method because the depth histogram (Figure 3) distributed $25 \%$ of the animats below the surface duct. Note, if animat \#2 were to stay inside the surface duct, then the two methods would arrive at the same result. Therefore, it is possible for the animat method to produce similar results to the static distribution method in some scenarios.

Although this is a trivial example, it illustrates the essential reason for differences between the static and animat methods. Removal of the temporal component eliminates the potential for existence in more than one sub-volume and reduces the risk of encountering higher level exposures. Intuitively, this becomes clear if we imagine a sound source ensonifying a small region with an SPL near $195 \mathrm{~dB}$ for an very long period of time. The animat method would reveal that (given enough time) eventually all of the marine mammals would pass through this high intensity region and be harassed. The static distribution method, on the other hand, would arrive at a smaller, fixed number of harassments based only on the typical volume distribution of the marine mammals. In section 4, more realistic scenarios are given to further illustrate this point.

\section{Theory/calculation}

In this section we discuss the elements needed to create a simulated underwater environment and calculate the acoustic transmission loss from a hypothetical source to points in that environment. The sound pressure levels are then applied via either the animat or static distribution method to determine the total number of marine mammal harassments that occur in the simulation space over the duration of the exercise.

Calculation of harassment numbers depends on three components:

1. Acoustic source

2. Propagation loss (or transmission loss)

3. Marine mammal distributions and harassment thresholds

We review the significant parameters for each of these components and choose specific values to define three different simulation scenarios.

\subsection{Acoustic source}

As discussed in the introduction, MFA sonars have become an acoustic source of interest in recent years and will therefore be used loosely as the basis for a hypothetical acoustic source in all subsequent simulations in this article. In particular, we will consider a simplified and idealized hull-mounted surface ship sonar with nominal source levels similar to the AN/SQS-53.

A number of sound source characteristics must be considered in an acoustic simulation. First, the source will have some defined beam pattern that describes the directionality of the emitted sound. The waveform of the outgoing signal, its amplitude, duration and duty cycle must be known to properly characterize the sound field. For the simulations in this article we will consider a hypothetical MFA sonar with a SL of $235 \mathrm{~dB}$ and an omni-directional beam pattern. It is assumed to be hull-mounted from a surface ship with an effective source depth 10 meters below the ocean surface. It is also assumed that the sonar emits each ping at a constant frequency of $3 \mathrm{kHz}$; harmonic components of the transmissions will not be considered. The ping duration is $1 \mathrm{~s}$ and is repeated once every $30 \mathrm{~s}$ for a total of 120 pings/hr. Finally, it is assumed that the ship remains stationary at the center of the simulation space.

\subsection{Propagation model}

The propagation model used to predict the acoustic exposure is, in general, a complicated function of many factors including the water temperature, pressure and salinity, as well as the sediment characteristics, and reflections from both the bathymetry and sea surface. The sound speed profile (SSP) indicates how the sound speed changes with increasing water depth and 
can be measured at a specific location (coordinates). In a given region, the SSP may be range-dependent and could change with season and possibly even time of day. Reflections from the sea floor and sea surface also affect acoustic propagation.

Even in a simplified environment with a flat bathymetry and calm sea surface, multipath reflections will sum (constructively or destructively) at various points in the water column. These interference effects create a spatially varying sound field. To capture the average sound field we calculate the incoherent transmission loss (Jensen et al., 2000).

In more complicated scenarios reflections from the sea floor will depend on bathymetry as well as the sediment materials and the thickness of each sediment layer. Similarly, reflections from the sea surface will depend on the sea state. Winds above the surface can generate ocean waves which in turn scatter the underwater acoustic waves. While these are important factors in determining the exact characteristics of the sound field they are less important for this analysis since we are comparing methods and will use the same sound field with each method being examined.

To evaluate and compare analysis methods we consider a square box ocean environment with a completely flat bathymetry and perfectly calm sea surface. The seabed is assumed to be a silty-sand with sound speed of $1550 \mathrm{~m} / \mathrm{s}$, density of $1.5 \mathrm{~g} / \mathrm{cm}^{3}$ and attenuation factor of $0.2 \mathrm{~dB}$ /wavelength.

The Bellhop Gaussian Beam propagation model was used to calculate the sound field and intensity for the sound source described above (Porter, 1987; Jensen et al., 2000). The pressure values were calculated every 5 meters in range and 5 meters in depth. Since the bottom of the ocean environment is always assumed to be flat, it is only necessary to calculate the transmission loss along a single transect, which can then be applied in at any bearing. The box ocean environments to be considered in this article extend $400 \mathrm{~km} \times 400 \mathrm{~km}$, with a depth of either $200 \mathrm{~m}$ or $5000 \mathrm{~m}$. To calculate the transmission loss at the corners of the box would require a maximum range of $282 \mathrm{~km}(200 \mathrm{~km} \times \sqrt{2})$; therefore, the sound intensity was calculated out to a maximum range of $300 \mathrm{~km}$, for each of the box ocean environments.

\subsection{Marine mammal simulations}

Two "synthetic" odontocetes were used for this study; one deep-diving species and one shallow-diving species. Each species was assumed to have the same uniform population density of 0.0025 mammals $/ \mathrm{km}^{2}$ and the species models were created using $3 \mathrm{MB}$

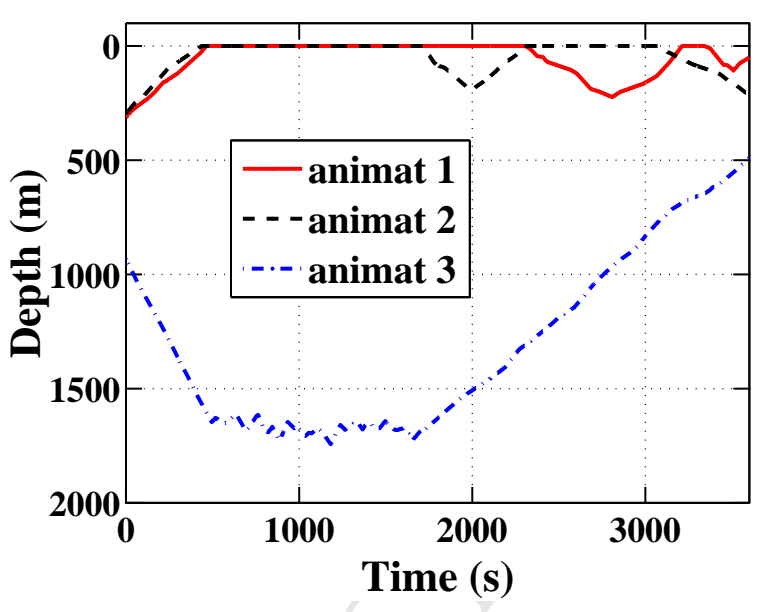

Figure 4: Simulated (vertical) dive pattern for three deep-diving animats during a one hour period. Horizontal motion (not shown) is also included in the simulated dive path of each animat.

software (Houser, 2006). The 3MB software was then used to simulate the swim and dive patterns of each of the animats for a period of 1 hour. Figure 4 shows an example of three animat tracks for a deep-diving species. Horizontal motion (not shown) is also included in the simulated dive path of each animat. The animat locations (coordinates and depth) were then exported at 30-s intervals to correspond with the emission times of the hypothetical sonar pings.

\section{Results and discussion}

In this section we will use the described propagation and animat models to simulate three different scenarios. Section 4.1 considers a shallow-diving species in shallow water. In section 4.2 a deep water environment with both shallow and deep-diving species is evaluated. In each scenario, the number of harassments is calculated using both the static distribution and animat methods. All three scenarios will demonstrate the same fundamental difference between the two calculation methods.

The animat method described in section 3 was repeated 50 times to provide 50 realizations in a Monte Carlo analysis (i.e. the simulation was run 50 times with varying start conditions and dive profiles for each run). After each realization, the number of harassments was recorded along with a running average of the harassments. The depth histogram for the static distribution 
method was created by binning the depth coordinates of all of the animats in all 50 realizations.

\subsection{Shallow Ocean}

In the first scenario we consider the box ocean, with dimensions $400 \mathrm{~km} \times 400 \mathrm{~km}$ and a depth of 200 meters. The sound speed profile is shown in panel A of Figure 5, and Bellhop was used to calculate the incoherent TL (Figure 5, panel B)from a $235 \mathrm{~dB}$ source at 5 meter increments in both depth and range.

Marine mammal movements from a shallow-diving marine mammal species were simulated using $3 \mathrm{MB}$ with 0.0025 animats $/ \mathrm{km}^{2}$ for a total of 400 animats. Four hundred animats with individually distinct dive patterns were exported from $3 \mathrm{MB}$ and evaluated by both the animat and static distribution methods. Coordinates and depths for each animat's path are extracted from the simulation and the maximum SPL for the overall dive profile is determined. The maximum received SPL value is applied to the risk function (Figure 1) to find the "risk" of that animat being harassed. Due to the randomized dive patterns of the animats in this scenario the simulation was repeated 50 times, and the number of harassments was evaluated for each realization. The number of harassments, $H_{i}$, for the $i$ th Monte Carlo realization is indicated by the black dashed line in Figure 6. The (estimated) mean number of harassments (red solid line) after $n$ realizations is

$$
\bar{H}_{n}=\frac{1}{n} \sum_{i=1}^{n} H_{i} .
$$

For each realization, the bars above and below the estimated mean indicate the estimated standard deviation, defined as

$$
\bar{\sigma}_{n}=\left(\frac{1}{n-1} \sum_{i=1}^{n}\left(H_{i}-\bar{H}_{n}\right)^{2}\right)^{\frac{1}{2}},
$$

Note that beyond 30 realizations, Figure 6 shows relatively little change in the estimated mean, $\bar{H}_{n}$, or standard deviation, $\bar{\sigma}_{n}$. When the number of realizations is large (more than 30) a confidence interval for the mean may be calculated by assuming the probability density function (pdf) of $H_{n}$ is Gaussian due to the central limit theorem. However, since the true variance of the distribution is unknown here, the pdf is assumed to be a Student's t-distribution and the estimated standard deviation is used to calculate the confidence interval with n-1 degrees of freedom, (Haldar and Mahadevan, 2000). Therefore, after 50 realizations there is $95 \%$

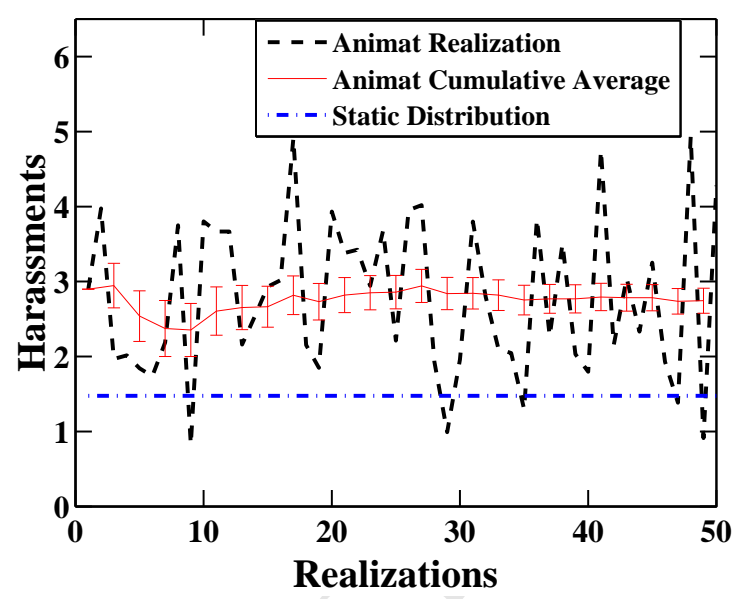

Figure 6: Number of harassments for a shallow-diving species in a shallow ocean environment (Figure 5). After 50 realizations the animat method results in a mean of 2.78 harassments. The bars indicate the standard deviation of the animat method. The static method, based on the histogram in Figure 7, arrives at a lower number of harassments.

confidence that the mean number of harassments for this scenario was, $\bar{H}_{50}=2.78 \pm 0.047$.

The ability to account for the variability in predictions resulting from variations in animat behavior is a capability of the animat approach which is not readily apparent when implementing the static distribution method. Predictions of variability need not be limited to the standard deviation and other statistical approaches could be implemented. Whatever estimate of variability is used, the stabilization of the measure across multiple runs can be used to limit the number of realizations (or iterations) in the Monte Carlo simulation. Conversely, keeping record of the number of harassments across individual realizations permits the probability of maximum harassment events to be estimated. In combination with a measure of variability, both an estimate of the range of expected harassments and the potential for "spurious events" can be made, i.e. events that have low probability of occurrence but a high number of takes, as functionally defined by the user. Such information would be beneficial to quantifying the uncertainty in impact estimates involving marine mammals and anthropogenic sound.

For the static distribution method, the random dive patterns from all 20,000 animats (400 animats $\times 50$ 
A

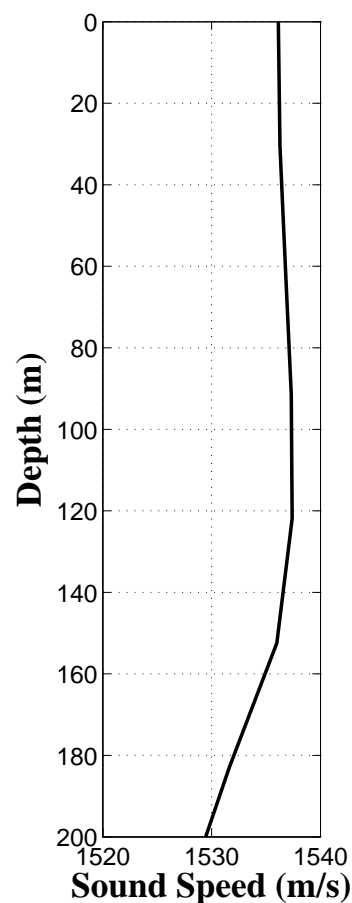

B

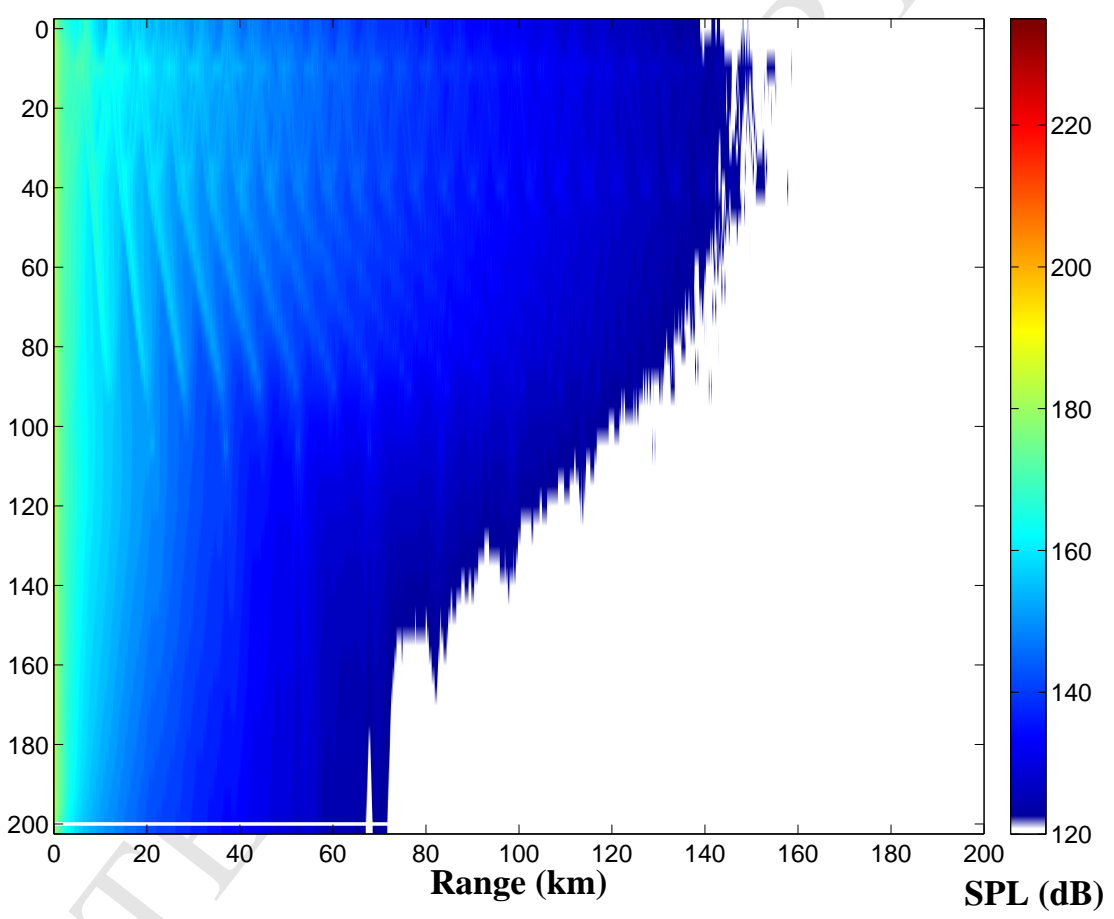

Figure 5: Panel A shows the Sound Speed Profile (SSP) for a 200 meter deep ocean environment. Panel B shows the sound pressure field (in dB SPL) produced by a source (located at $0 \mathrm{~km}$ in range and $10 \mathrm{~m} \mathrm{depth).} \mathrm{Sound}$ pressure levels are mapped to the risk function (Figure 1) for calculation of harassments in both the animat and static distribution analyses. SPL values below $120 \mathrm{~dB}$ are not displayed here because the risk goes to zero. 


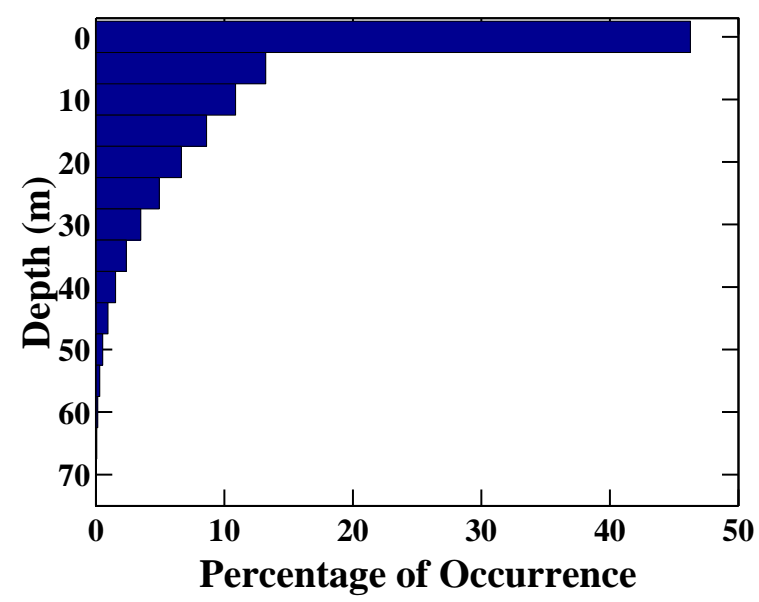

Figure 7: The depth histogram for a shallow-diving species is used to calculate harassments in the static distribution method.

realizations) were used to calculate the depth histogram shown in Figure 7. The $400 \mathrm{~km} \times 400 \mathrm{~km}$ ocean surface was divided into $500 \mathrm{~m} \times 500 \mathrm{~m}$ cells for a total of 40,000 cells. The model then applied the same animat distribution of 0.0025 animats $/ \mathrm{km}^{2}$ to each of the cells (which have an area of $0.25 \mathrm{~km}^{2}$ ) to get $6.25 \times 10^{-4}$ animats per cell. In the column below each cell, the $6.25 \times 10^{-4}$ animats were distributed according to the depth histogram in Figure 7. The proportion is applied to the population distribution to arrive at the number of harassments for every column. Summing all of the harassments from all of the columns gives the total number of harassments in the simulation space. The resulting number of harassments is plotted in Figure 6 for comparison with estimates obtained from the animat method. The number of harassments from each analysis method is also listed in Table 1.

Note that in this example the static distribution method arrived at 1.48 harassments which was $46.8 \%$ lower than the mean harassment estimate obtained by the animat method. In fact, the static distribution estimate is below the lower limit of the $95 \%$ confidence interval for the animat method $(2.78-0.047=2.733)$. Although in at least one of the 50 realizations the harassment estimate was similar to that obtained with the static distribution method, the static distribution method consistently underestimated the harassments obtained with the animat method.

\subsection{Deep Ocean}

In this section, another $400 \mathrm{~km} \times 400 \mathrm{~km}$ box ocean environment is considered, but with a depth of $5 \mathrm{~km}$. Bellhop was used to calculate the incoherent TL from a $235 \mathrm{~dB}$ source at 5 meter increments in both depth and range. The SSP is shown in panel A of Figure 8. Note that a "Munk" profile was used. This is a canonical sound speed profile used for deep water scenarios and produces the convergence zones every $65 \mathrm{~km}$ in range from the source, as seen in panel B of Figure 8.

Marine mammal movements were simulated using $3 \mathrm{MB}$ for the same shallow-diving species that was considered in the previous section. The simulation was repeated 50 times, and the number of harassments was evaluated for each realization. The average number of harassments is plotted (red solid line) in Figure 9, where the bars indicate the total standard deviation after each realization. After 50 realizations there is $95 \%$ confidence that the mean number of harassments for this scenario was, $\bar{H}_{50}=0.155 \pm 0.00916$. The depth histogram for the shallow-diving species was identical to the one in the shallow ocean case (Figure 7) because this species does not dive below $100 \mathrm{~m}$. The number of harassments resulting from the static distribution method (0.0194) is listed in Table 1 and plotted in Figure 9 for comparison with the animat method. Note that the static distribution method produced a result that was lower than the animat method.

Finally, a deep-diving animat species was evaluated in this deeper ocean environment. Again, the animat analysis was repeated for 50 realizations, and the average number of harassments after each realization is plotted in Figure 10. After 50 realizations there is 95\% confidence that the mean number of harassments for this scenario was, $\bar{H}_{50}=0.331 \pm 0.0679$. For the static distribution method the depth histogram shown in Figure 11 was created based on the dive patterns of all 20,000 deep-diving animats. Results of the static distribution analysis are plotted in Figure 10 and listed in Table 1 for comparison with the animat method. The number of harassments in the static distribution method is less than half that of the animat method.

\section{Conclusion}

Multiple methods of estimating the impact to marine mammals exposed to anthropogenic sound exist. In this manuscript we compare two methods which have previously formed the basis of EIS's created by the US Navy; 
Table 1: Number of harassments in a $400 \mathrm{~km} \times 400 \mathrm{~km}$ box ocean environment. The number of harassments in the static distribution method due to the histogram distributions in Figures 7 and 11 are lower than the harassments from the animat method with animats moving for 1 hour.

\begin{tabular}{ccccc}
\hline Ocean Depth & Species & Animat method & Static distribution method & $\%$ difference \\
\hline $200 \mathrm{~m}$ & Shallow-diving & 2.78 & 1.48 & $46.8 \%$ \\
$5 \mathrm{~km}$ & Shallow-diving & 0.155 & 0.0194 & $87.7 \%$ \\
$5 \mathrm{~km}$ & Deep-diving & 0.331 & 0.0484 & $85.5 \%$ \\
\hline
\end{tabular}
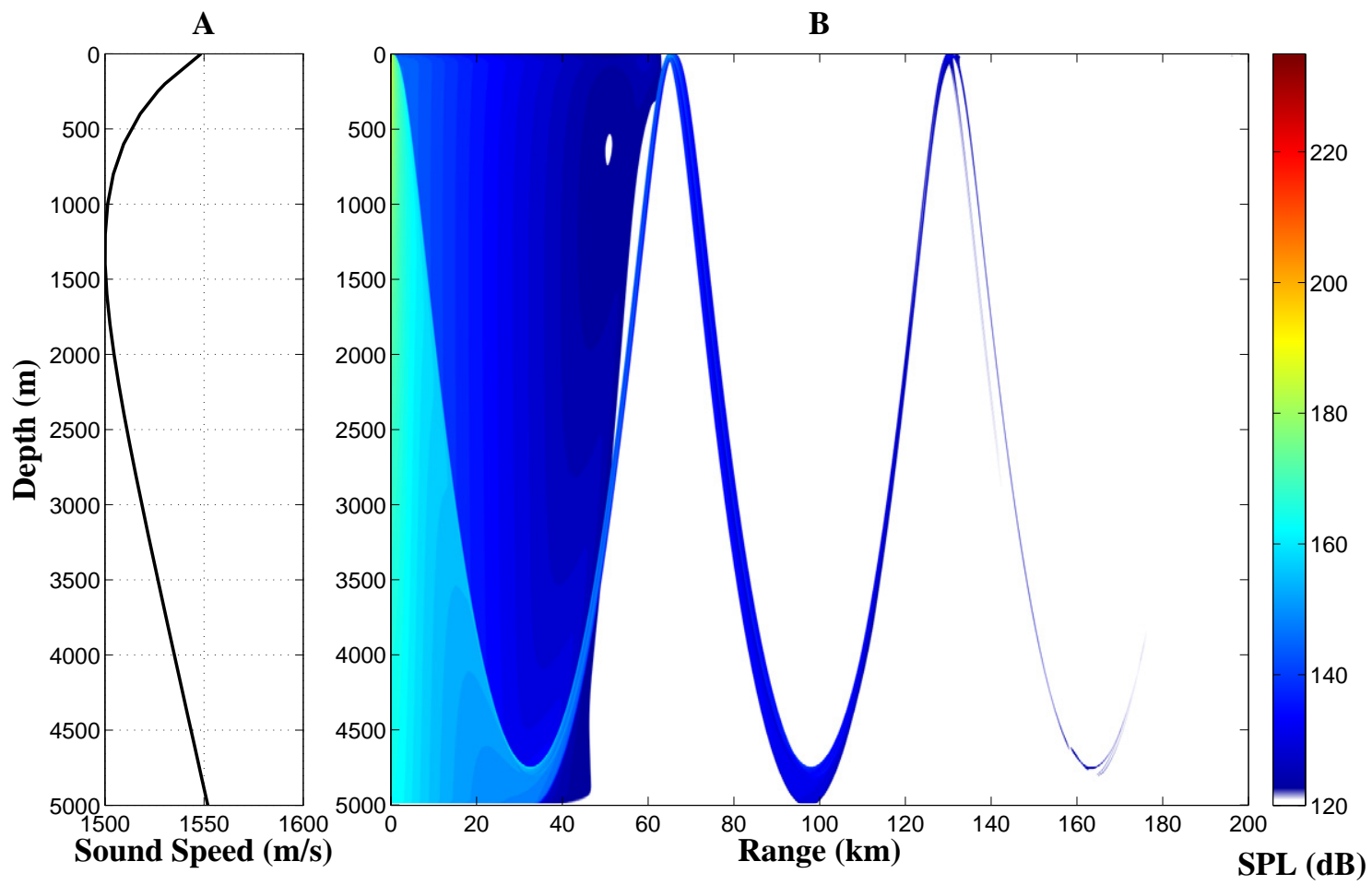

Figure 8: Panel A shows the Sound Speed Profile (SSP) for typical $5 \mathrm{~km}$ deep ocean environment. Panel B shows the sound pressure field (dB SPL) resulting from a source (located at $0 \mathrm{~km}$ in range and $10 \mathrm{~m}$ depth). Sound pressure levels are mapped to the risk function (Figure 1) for calculation of harassments in both the animat and static distribution analyses. SPL values below $120 \mathrm{~dB}$ are not displayed here because the risk goes to zero. 
A

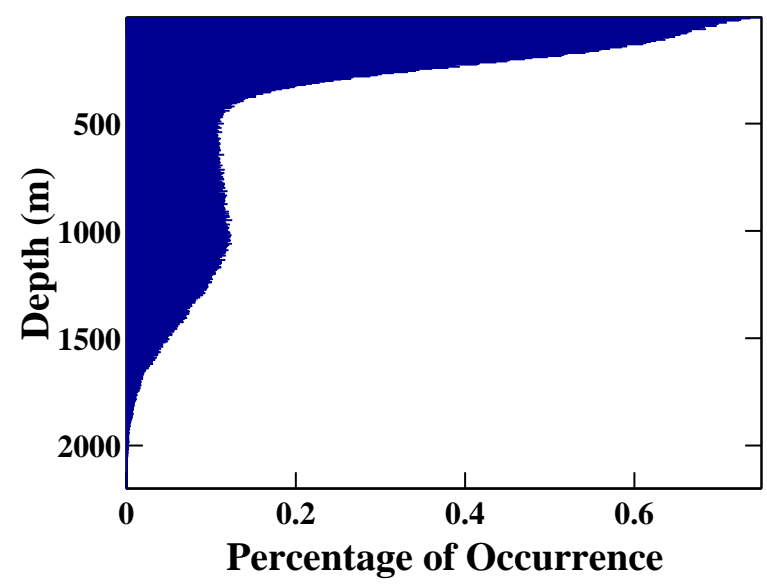

B

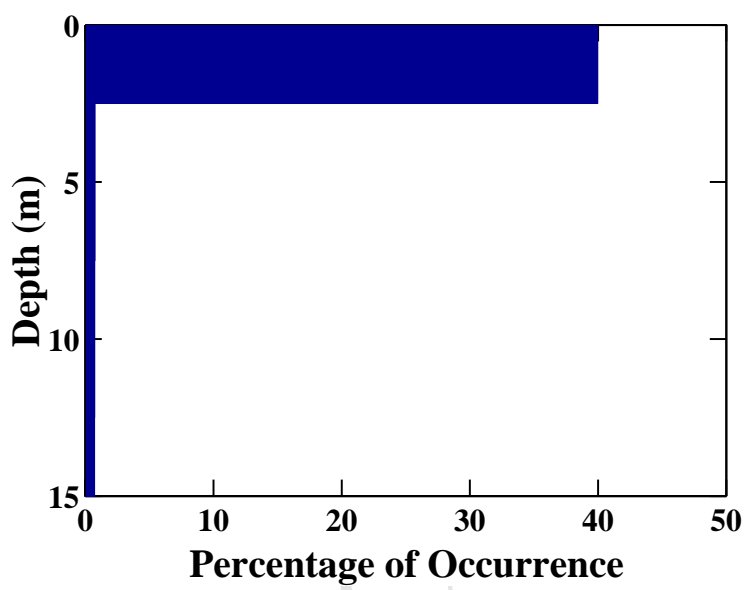

Figure 11: Panel A. The depth histogram for a deep-diving species in a $5 \mathrm{~km}$ deep ocean environment (Figure 8) is used to calculate harassments in the static distribution method. Panel B shows an expanded view of the first 15 meters below the surface.

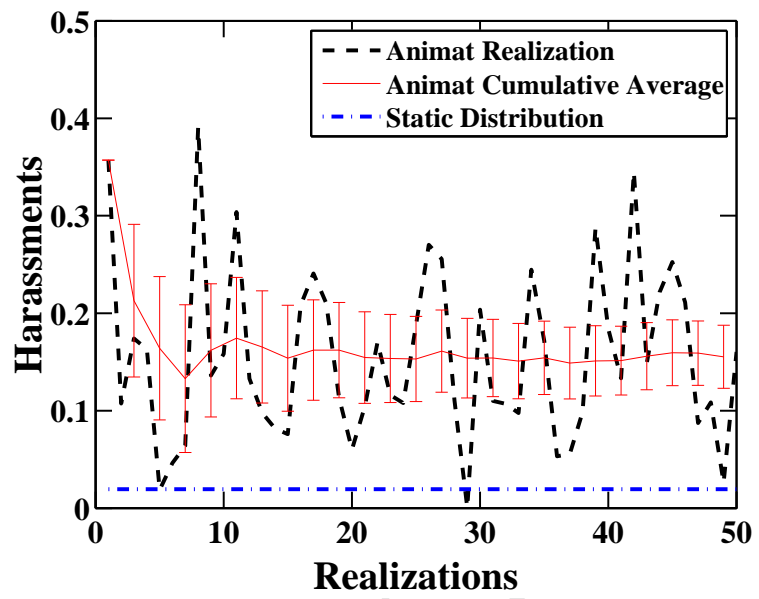

Figure 9: Number of harassments for a shallow-diving species in deep ocean environment (Figure 8). After 50 realizations the animat method results in a mean of 0.155 harassments. The bars indicate the standard deviation of the animat method. The static method, based on the histogram in Figure 7, arrives at a lower number of harassments.

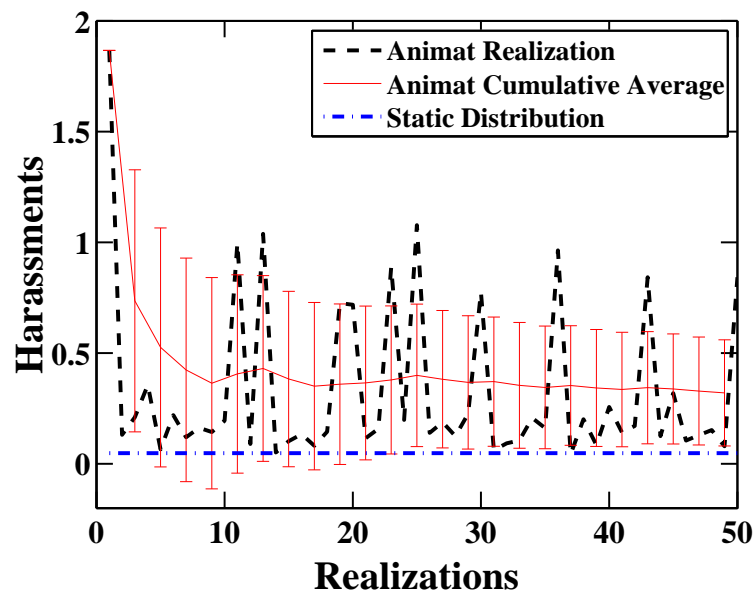

Figure 10: Number of harassments for a deep-diving species in a deep ocean environment (Figure 8). After 50 realizations the animat method results in a mean of 0.331 harassments. The bars indicate the standard deviation of the animat method. The static method, based on the histogram in Figure 11, arrives at a lower number of harassments. 
the static distribution method and the animat method. The two approaches both calculate behavioral harassments of marine mammals based on the calculated maximum received level of sound (in SPL) and the application of this value to a risk function. The static distribution method is intuitively straightforward and reduces the complexity of the problem to a 3D space. The animat method is more computationally intensive, but is more realistic in its treatment of marine mammal exposures to sound in time and space (i.e. a 4D problem).

The exercises presented here demonstrate that the static distribution method of estimating Level B harassment of marine mammals consistently produces lower estimates than those produced by the animat method. Fundamentally, this occurs because the static distribution method forgoes the $4^{\text {th }}$ dimension of the problem. It does not account for the occupancy of more than one sub-volume of ocean by individual animals. Marine mammals are dynamic in time and space and can traverse ranges and depths during military exercises or industrial activities that move them through multiple subvolumes with differing levels of sound exposure. Although distributing animals in space is an idealized and intuitive approach to estimating sound exposure within a population of marine mammals existing in a region of anthropogenic acoustic activity, marine mammals are not "fractional." Implementation of marine mammals as distinct and individual entities in impact estimates provides a better approximation of the "real world" by accounting for the behaviors of individual animals in time and space.

The animat method additionally offers some benefits to the quantification of uncertainty. First, the ability to calculate variability in harassment estimates can provide regulators with better understanding of the potential impact to marine species. Second, the ability to determine a probability of a "spurious event," in which many marine mammal harassments may occur, can be calculated. Collectively, these pieces of information will better inform the regulator as to the potential for impact to marine mammals resulting from the introduction of sound into the ocean by human-kind.

\section{References}

Cox, T. M., Ragen, T. J., Read, A. J., Vos, E., Baird, R. W., Balcomb, K., Barlow, J., Caldwell, J., Cranford, T., Crum, L. et al. (2006). Understanding the impacts of anthropogenic sound on beaked whales. Journal of Cetacean Research and Management 7, 177-187.

D’Amico, A., Gisiner, R. C., Kettern, D. R., Hammock, J. A., Johnson, C., Tyack, P. L. and Mead, J. 2009. "Beaked whale strandings and naval exercises," Aquatic Mammals, 35(4): 452-472.
DeAngelis, D. L. and Gross, L. J. 1992. Individual-Based Models and Approaches in Ecology. Chapman and Hall, New York.

Fernandez, A., Edwards, J., Martin, V., Rodriguez, F., Espinosa de los Monteros, A., Herraez, P., Castro, P., Jaber, J. R. and Arbelo, M. (2005). "Gas and fat embolic syndrome" involving a mass stranding of beaked whales exposed to anthropogenic sonar signals. Journal of Veterinary Pathology 42, 446-457.

Frankel, A. S., Ellison, W. T. and Buchanan J., "Application of the acoustic integration model (AIM) to predict and minimize environmental impacts," in Proc. Oceans 2002, 2002, pp. 1438-1443.

Frantzis, A. (1998). Does acoustic testing strand whales? In Nature, vol. 392 , pp. 29

Haldar, A., Mahadevan, S., 2000. Probability, Reliability and Statistical Methods in Engineering Design, pp. 126-129, John Wiley and Sons, New York.

Houser, D. S. (2006). A method for modeling marine mammal movement and behavior for environmental impact assessment. IEEE Journal of Oceanic Engineering 31, 76-81.

Jensen, F.B., Kuperman, W.A., Porter,M.B., and Schmidt, H., 2000. Computational Ocean Acoustics. Springer-Verlag, New York.

Jepson, P. D., Arbelo, M., Deaville, R., Patterson, I. A. R., Castro, P., Baker, J. R., Degollada, E., Ross, H. M., Herrez, P., Pocknell, A. M. et al. (2003). Gas-bubble lesions in stranded cetaceans. Nature $425,575-576$.

National Research Council (NRC). (2005). Marine mammal populations and ocean noise. Washington, DC: National Academies Press.

Porter, M.B., 1987. Gaussian beam tracing for computing ocean acoustic fields. Journal of the Acoustic Society of America 82, 4, 1349-1359.

Rommel, S. A., Costidis, A. M., Fernandez, A., Jepson, P. D., Pabst, D. A., McLellan, W. A., Houser, D. S., Cranford, T. W., Helden, A. L. V., Aleen, D. M. et al. (2006). Elements of beaked whale anatomy and diving physiology and some hypothetical causes of sonar-related stranding. Journal of Cetacean Research and Management 7, 189-209.

Simmonds, M. P. and Lopez-Jurado, L. F. (1991). Whales and the military. Nature $351,448$.

Urick, R. J., 1975. Principles of Underwater Sound, second ed. McGraw-Hill, New York.

U.S. Department of Commerce and U.S. Department of the Navy. (2001). Joint Interim Report Bahamas Marine Mammal Stranding Event of 14-16 March 2000. Washington, DC: Department of Commerce.

U.S. Department of the Navy. (1998). Final Environmental Impact Statement, Shock-testing the SEAWOLF submarine. Washington, DC: Department of the Navy.

U.S. Department of the Navy. (2001). Final Environmental Impact Statement for Surveillance Towed Array Sensor System Lowfrequency Active (SURTASS LFA) Sonar. Washington, DC: Department of the Navy.

U.S. Department of the Navy. (2005). Draft Overseas Environmental Impact Statement/Environmental Impact Statement, Undersea Warfare Training Range. Washington, DC: Department of the Navy.

U.S. Department of the Navy. (2008). Hawaii Range Complex, Final Environmental Impact Statement/Overseas Environmental Impact Statement. Washington, DC: Department of the Navy. 
- We compare two analysis methods to estimate the impact of sound on marine mammals.

- Differences in the number of behavioral harassments from each method are assessed.

- The static distribution method assumes a fixed spatial distribution of mammals.

- The animat method assumes dynamic mammals moving in space and time.

- The static distribution method underestimates harassment relative to the animat method. 